\title{
High Throughput Centrifugal Adhesion Screening Test for Soft Materials
}

\author{
Yusu Chen ${ }^{\dagger}$, Qifeng Wang ${ }^{\ddagger}$, Carolyn E. Mills ${ }^{\dagger}$, Johanna G. Kann ${ }^{\dagger}$, Kenneth R. Shull*, ${ }^{*}$, Danielle Tullman-
} Ercek $^{*, \dagger}$, Muzhou Wang*, ${ }^{*}$

'Department of Chemical and Biological Engineering, Northwestern University, 2145 Sheridan Road, Technological Institute, Evanston, Illinois 60208-3120, United States

\begin{abstract}
High-throughput screening of material mechanical properties has the potential to transform material science research in both aiding in material discovery and developing predictive models. However, the development of these assays is inherently difficult with only a few methods and tools reported, and the mounting demand for enormous material property datasets to develop predictive models is unfulfilled by the limited throughput of the current techniques. In particular, equipment cost and instrument limitations prohibit the widespread generation of large material property datasets. We address this problem by developing a high-throughput colorimetric method for testing mechanical adhesion using a common laboratory centrifuge, multi-well plates and microparticles. The technique uses centrifugation to apply a homogenous mechanical detachment force across the samples in the multi-well plate. We also develop a high-throughput sample deposition method to prepare films with uniform thickness in each well, minimizing well-to-well variability in measurements. Our centrifugal adhesion testing method can differentiate polymer films with variate adhesion strengths and shows excellent agreement with the probe tack adhesion test. To illustrate the throughput and consistency of the overall process, we displayed a pattern on a multi-well plate by depositing two different formulations and performing the centrifugal test. We can achieve a throughput of thousands of samples per run, and it is limited only by the number of wells in the plates. With its simplicity, low cost and large dynamic range, this highthroughput method has the potential to change the landscape of adhesive material characterization.
\end{abstract}

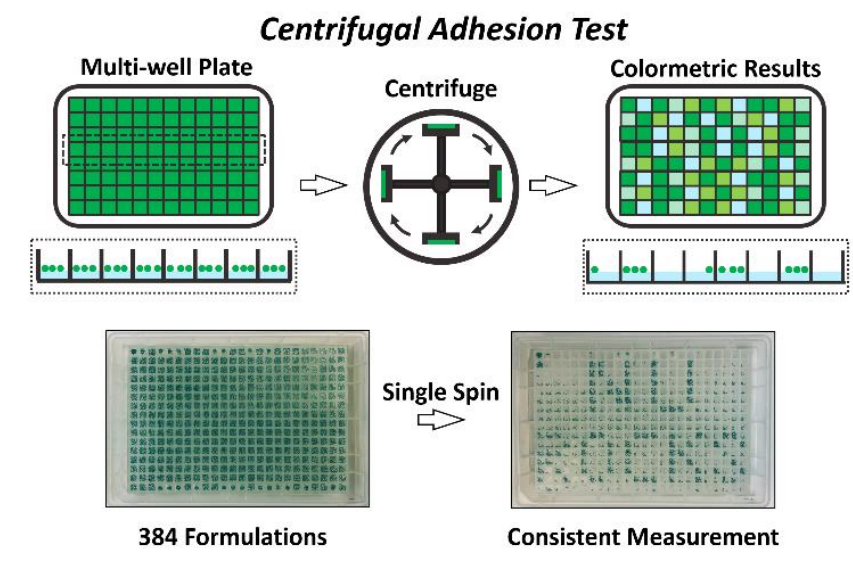

\section{Introduction}

Advances in combinatorial chemistry, synthetic biology, and sequence-specific polymers have drastically increased the sizes of materials libraries for applications ranging from nanomedicines to catalysts ${ }^{1-3}$. Advanced computational tools and machine learning have matched this expanded capability by 
training models for predictive design using these large data sets ${ }^{1,4,5}$. Characterization techniques also have the potential to provide data at a rate to assess all of these new materials. For example, biological properties such as enzyme activity and antibody binding are routinely tested at a rate of $10^{5}-10^{6}$ per day ${ }^{6,7}$ by utilizing colorimetric or fluorescence assays. However, techniques to characterize properties outside the biological realm have yet to match the required throughput for the exponentially increasing demand for new experimental data, particularly those for mechanical properties such as adhesion. A key challenge to reaching a similar throughput with mechanical testing is the low throughput of common, existing techniques for both sample preparation and measurement. A successful rapid, cost-effective, and straightforward high-throughput characterization pipeline would expedite both the development of predictive models and the discovery of novel materials. The ability to implement such a pipeline with standard lab equipment would ensure accessibility to the materials community at large.

Many techniques have been developed to reach a higher throughput for mechanical testing to enable combinatorial material design ${ }^{8}$. Existing methods can measure properties such as elastic modulus, hardness, and residual stress at throughputs of hundreds or thousands of formulations per run. These methods use techniques such as scanning nanoindentation, micromachined cantilever beams, and micro-electromechanical systems ${ }^{9-11}$. However, these tests require the samples to be fabricated in a specific form or special custom-made instrumentation ${ }^{12,13}$. As a result, either the low throughput of the specific fabrication or instrumentation requirements significantly hinder the accessibility and the overall throughput of these techniques. Therefore, a high-throughput mechanical testing pipeline that combines simple sample fabrication/preparation steps with easy characterization techniques would provide the materials science community broad access to large mechanical property datasets that enable transformative advances.

Adhesion phenomena are essential in compliant materials with low elastic moduli, a category widely encompassing biological soft tissues, elastomers, and hydrogels, etc. ${ }^{14}$. Specifically, soft matter adhesion phenomena are crucial in various medical and industrial applications, such as bioimplants, sealing agents, and ship fouling prevention ${ }^{15,16}$. In the case of medical adhesives, such as tissue sealants, exceptional underwater adhesion is required to prevent blood loss and possible infections ${ }^{17}$. Adhesives inspired by biological organisms such as geckos and mussels are the promising candidates that offer excellent reversible adhesive properties in both dry and wet environments ${ }^{18,19}$. Achieving these desired adhesive properties depends on the ability to finely tune adhesive strength via several iterations of formulation and characterization, which can be costly and time-consuming. Thus, a characterization method that can quantitively measure adhesive strength in a high-throughput and cost-effective manner is clearly necessary to accelerate the development of novel adhesive materials.

High-throughput adhesion testing often requires preparing samples in the particular form of gradients or arrays, which frequently involves building complex custom-made instrumentation. For example, Potyrailo et al. developed a high-throughput adhesion loss characterization method that can characterize coating materials at a 10-fold increase in throughput from 5 coatings per day up to 48 coatings per day, but this method requires a complex automated system that complicates widespread adoption. Other characterization methods have also been repurposed to measure adhesion in relatively high throughput. For example, atomic force microscopy (AFM) can determine the adhesion force on the surface by measuring the deformation of the cantilever, but it can only measure one sample per run and requires expensive instrumentation. Although the automation of AFM measurements potentially gives a higher throughput ${ }^{20,21}$, the expensive instrumentation prevents the method from being widely accessible.

We identified three key requirements for a high-throughput adhesion characterization pipeline to be widely adopted: (1) it uses commonly available laboratory equipment, (2) it applies the same force to many 
samples simultaneously, and (3) it efficiently converts a mechanical response into an optical signal. A common laboratory centrifuge satisfies the first two criteria and is used in assessments of cell adhesion and biophysical properties. Reyes et al. reported using centrifugation in quantitative measurements of cell adhesion, allowing for fast screening of biomaterial surface modifications and tissue scaffolds 22 . Centrifugation has also been used to mechanically manipulate single molecules and enable the measure of the rupture and unlooping force of DNA nanoswitches in high throughput with high precision ${ }^{23,24}$. Inspired by these examples, we propose a centrifuge-based high-throughput mechanical testing workflow. We were also inspired by the well-known probe tack apparatus used to measure the adhesion energy of material between a spherical probe and a flat surface ${ }^{25}$. By replacing the spherical probe with colored or fluorescent spherical particles and centrifuging, we can correlate adhesive strength to particle retention at a known centrifugal force, which is easily visualized and thus satisfies requirement (3) (Fig. 1).
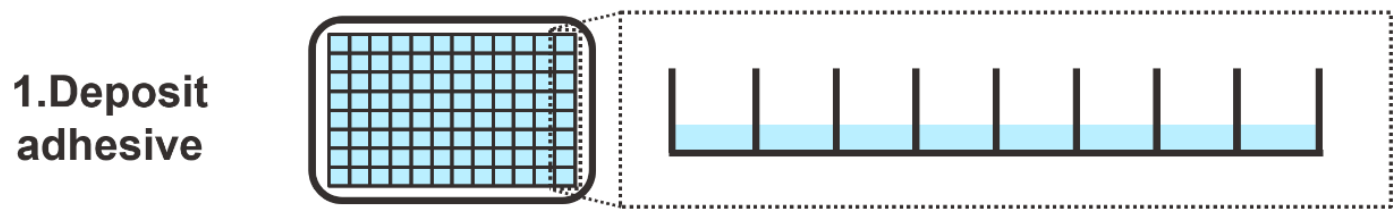

\section{Apply microspheres}
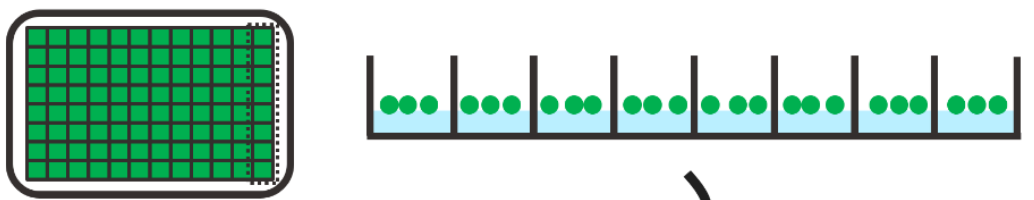

\section{Centrifugal testing}

\section{Comparative measurement}
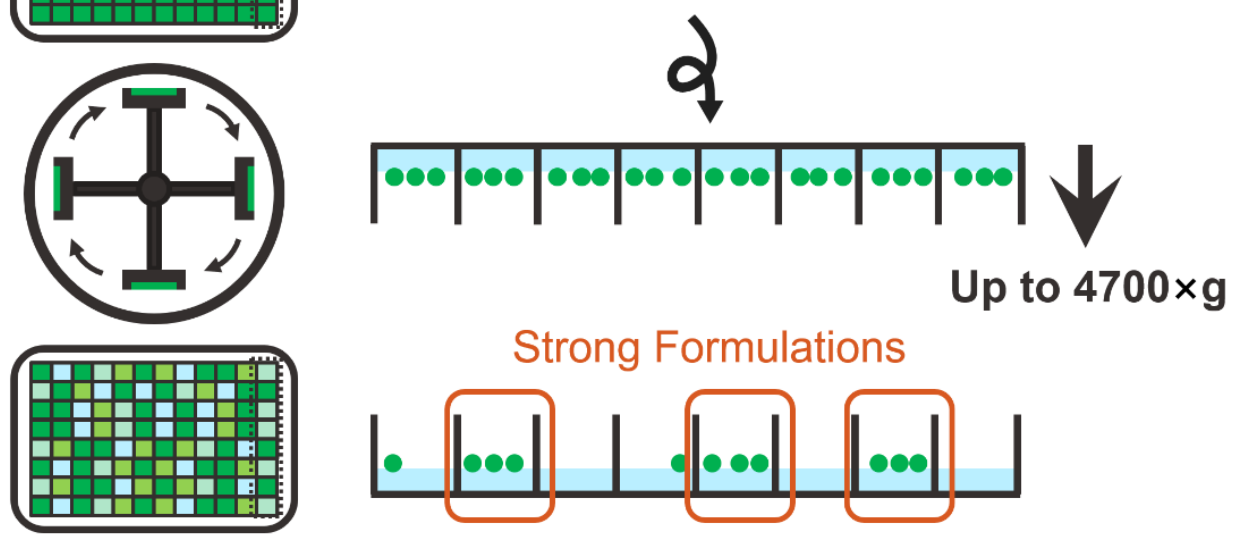

Figure 1. Schematic of the centrifugal adhesion testing procedure. After casting adhesive films in a multi-well plate, colored or fluorescent particles are evenly distributed in each of the wells, then the plate is centrifuged with the particles facing outward, causing the particles to detach from the films with weaker adhesive properties. Arrow indicates the direction of centrifugal acceleration (up to $4700 \times \mathrm{g}$ ).

In this article, we describe a novel pipeline that can prepare uniform thickness films with individual formulation and measure adhesive properties at high throughput with a common benchtop centrifuge, microparticles, and commercially available multi-well plates. The method can characterize thousands of formulations simultaneously. We demonstrate the method with a model pressure sensitive adhesive (PSA) polymer and calibrate our characterization method to the measurements made by a standard probe tack test with excellent agreement. The simplicity of our method can speed up the characterization process of new adhesive materials by a throughput of 1536 samples per run, $\sim 1000$ times higher than the traditional onesample-per-run method. 


\section{Experimental Section}

PSA film preparation. We demonstrated our method using a waterborne acrylic PSA (Figure S1) that is a copolymer of n-butyl acrylate with 3\% of methacrylic acid (Dow Chemical). The provided polymer solution is emulsion-based with a solid content of $\sim 40 \mathrm{wt} \%$. To reduce the viscosity of the solution, we prepared a $6.25 \mathrm{wt} \%$ working stock PSA solution by diluting with $\mathrm{ddH}_{2} \mathrm{O}$ to facilitate pipetting. Additionally, we prepared a $6.25 \mathrm{wt} \%$ solution of an inherently non-adhesive polymer, polyethylene glycol (PEG, $\mathrm{Mn}=300$ $\mathrm{g} / \mathrm{mol}$ ), in water. By mixing the two solutions in different ratios, we made working stocks of the polymer mixture at varying concentrations (70-100\% of PSA). We then pipetted $5 \mu \mathrm{L}$ total of the polymer solution with different formulations into corresponding wells of a 384-well plate (Nunc ${ }^{\mathrm{TM}}$ 384-Well, Non-Treated, Flat-Bottom Microplate, Thermo Scientific ${ }^{\mathrm{TM}}$ ). Using a benchtop centrifuge (Sorvall ${ }^{\mathrm{TM}}$ Legend $^{\mathrm{TM}}$ XFR Centrifuge with a TX-750 Swinging Bucket Rotor, Thermo Scientific ${ }^{\mathrm{TM}}$ ), the plate was spun at $1000 \times \mathrm{g}, 40^{\circ} \mathrm{C}$ for six hours to evaporate the water in the polymer solution. To ensure the films were thoroughly dried, we placed the 384-well plate in a vacuum oven at $65^{\circ} \mathrm{C}$ for one hour to further evaporate the water. We then characterized the morphology and the thickness of the films using a Contour X-100 3D optical profilometer (Bruker).

In situ bottom layer curing To cast a bottom layer in each well of the multi-well plate, we adapted the formulation and the procedure from Rapp et al. ${ }^{26}$. We chose polymethyl methacrylate (PMMA) as it is fast curing and has a glass transition temperature much higher than room temperature ${ }^{27}$. We made a prepolymer of PMMA by dissolving PMMA (purchased from Sigma Aldrich, average Mw 15,000 by GPC) in methyl methacrylate (Fisher Chemical) at a 1:2 mass ratio. In an amber vial, we added 2-hydroxy-2methylpropiophenone as the initiator (5\%), ethylene glycol dimethacrylate as the crosslinker (5\%) and prepolymer (90\%) and sparged the solution with nitrogen for 1 hour. We then pipetted $30 \mu \mathrm{L}$ of the mixed solution into each well of a 384-well plate (384PP 2.0, Labcyte). To cure the polymers while centrifuging, we purchased a UV LED light panel (UV intensity $0.1 \mathrm{~mW} / \mathrm{cm}^{3}$, LED Cool Lights) and constructed a custom setup in the centrifuge rotor (Figure S2). The setup was then centrifuged $(3000 \times \mathrm{g}, 10$ hours) while the prepolymers were UV irradiated, and then placed in a vacuum oven at $65^{\circ} \mathrm{C}$ for 1 hour remove any residual uncured monomer. The morphology and the thickness profile were measured by a Contour X-100 3D optical profilometer.

Centrifugal adhesion test We purchased green silica microparticles with size ranges of 600-710 $\mu \mathrm{m}$ (avg. geometric radius $325 \mu \mathrm{m}$ ) and $710-850 \mu \mathrm{m}$ (avg. geometric radius $390 \mu \mathrm{m}$ ) in diameter from Corpuscular Inc. Microparticles were then sieved through three U.S. standard stainless steel sieves purchased from Fisher Scientific (pore sizes: $600 \mu \mathrm{m}, 710 \mu \mathrm{m}$ and $850 \mu \mathrm{m}$ ) to ensure their sizes were correct. Each of the PSA films was then covered with a layer of microparticles of a specific size range. We then centrifuged the 384-well plate containing the PSA films with the particles facing inward at $4700 \times \mathrm{g}$ for 5 minutes to ensure good particle contact with the film. A picture of the plate was taken as a reference. The plate was then flipped so that the particles face outward; the plate was centrifuged at various angular speeds for $1 \mathrm{~min}$. After each spin, we took a picture of the plate and counted the number of particles in each well. For the high-throughput assay, we instead used red fluorescent silica microparticles with a size range of 600-710 $\mu \mathrm{m}$ in diameter from Cospheric Inc. (exc. $575 \mathrm{~nm}$, em. $607 \mathrm{~nm}$ ). We followed the same procedure for the green silica particles to perform the centrifugal adhesion test. For characterization, we measured the fluorescence reading with a plate reader (Synergy ${ }^{\mathrm{TM}}$ HTX, Biotek) prior to and after spinning at each speed. 
The axisymmetric probe tack test We performed a probe tack test to measure the force vs. displacement history for each formulation under the same strain-rate. The setup was previously described by Wang et al. ${ }^{28}$. We used the same microparticle from the centrifugal test to replace the hemispherical glass indenter described in the paper. We first measured the contact area between the particle and the adhesive film during the centrifugal adhesion test for each formulation. To ensure consistency, we steadily increased the compressive load until the same contact area as the particle-film interface from the centrifugal method was achieved with an approaching velocity of $1 \mu \mathrm{m} / \mathrm{s}$ in the probe tack test. The contact area of the particle-film interface was monitored using the built-in microscope of the probe tack apparatus. We allowed a $1 \mathrm{~s}$ dwell time to improve the reproducibility of the measurement ${ }^{29}$. The indenter was then detached with the same velocity while measuring the detachment force. We obtained $\mathrm{F}_{\text {avg }}$ from the force vs. displacement profile by integrating the force-displacement curve and normalizing it by the total displacement, $\delta$ (Figure S3):

$$
F_{\text {avg }}=\frac{1}{\delta_{\text {total }}} \int F d \delta
$$

Plate image analysis and Z'-score calculation The image processing was performed with a Python script. Each multi-well plate image was converted to grayscale then split into a 24 by 16 grid, with each grid representing each well. For each grid box, a threshold value is then calculated to distinguish the particles, which are darker in color, from the background, which is lighter in color, of the well. The code then counts the number of pixels registered as particles and divides that value by the total pixel count of the grid box. The area ratios were calculated for all the wells on the control photo $(0 \times \mathrm{g})$, and the area ratios of each of the wells at different centrifugal speeds were divided by the ratios calculated in the control photo. The final ratios calculated represent the detachment of particles in each well. A screening window coefficient for high throughput screening assays, Z-factor, was used to quantify the assay signal dynamic range and the data measurement variation. A Z-score of 0.5-1.0 is usually considered excellent. The Z-score of the centrifugal adhesion method was calculated as previously described, with the $70 \%$ PSA as the negative control and the $100 \%$ PSA as the positive control ${ }^{30}$.

\section{Results and Discussion}

\section{Centrifugation enables film deposition with uniform thickness}

One of the significant challenges for realizing high-throughput mechanical testing is preparing samples consistently and combinatorially in a simple manner. To achieve this goal, we selected an emulsion-based acrylic pressure sensitive adhesive (PSA) polymer as the model material to demonstrate our sample preparation method. An accurate adhesion measurement relies heavily upon sample preparation, specifically ensuring the sample films have a uniform thickness, as adhesive properties are dependent on the film thickness ${ }^{31}$. Though drop-casting on a substrate is commonly used, this causes an uneven surface of the deposited film because of the "coffee-ring" effect from surface tension in which capillary flow drives a concentration gradient to the outer edge of a drying droplet (Figure 2.A and B) ${ }^{32}$. To solve this issue, we investigated the feasibility of film deposition while applying centrifugal force. We spun a 384-well plate in a centrifuge while drying the PSA solution in the plate for 6 hours. The water removal process was done under ambient ventilation at $40^{\circ} \mathrm{C}$, but the drying process can be significantly expedited if the polymer is in a more volatile solvent or the centrifuge can be heated at higher temperatures. The resulting films were transparent and smooth upon visual inspection. We later measured the morphology of the films using a 3D optical profilometer. The images showed the deposited films have a consistent thickness with a much smoother surface compared to films prepared by drop-casting (Figure 2.D). The measured thickness of $20 \mu \mathrm{m}$ matched 
the expected thickness calculated from mass and density (Table S1).

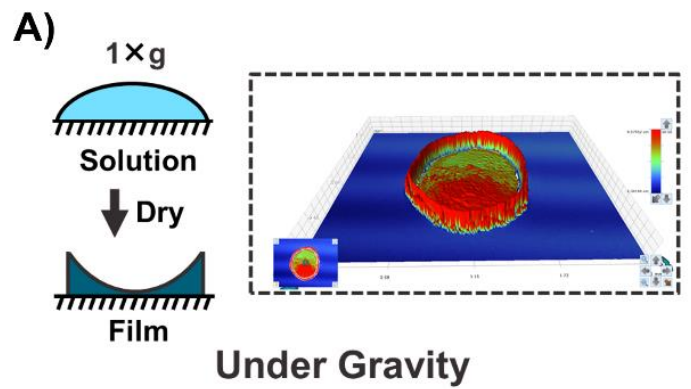

B)

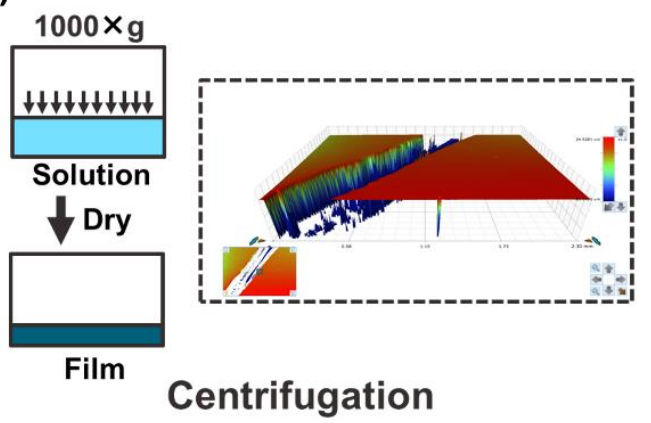

Figure 2. 3D optical profilometry of adhesive polymer films A) dried under gravity and B) under centrifugation. The film that was dried under centrifugation showed a significantly smoother surface than the one under gravity. The groove was purposely created before the measurement to obtain the thickness of the film.

The centrifugal film casting method introduces another challenge that arises from the finite size of the multi-well plate relative to the spinning radius of the centrifuge. Specifically, wells that are farther away from the center of the plate experience centrifugal forces that are misaligned relative to the bottom of the plate, resulting in slanted films of non-uniform thickness (Figure 3.A). To overcome this limitation, a hard bottom layer that is perpendicular to the centrifugal force at each well position can be cast prior to the film deposition step, using a similar centrifugal film casting method as discussed above. Thus, as the deposited films will slant at the same angle as the hard bottom layer, the relative thickness of the film deposited on the hard layer is uniform (Figure 3.B). We cast this hard bottom layer from polymethyl methacrylate by UV curing in situ while the plate was centrifuging ${ }^{26}$. We designed a custom-made in situ UV-curing system (Figure S2) and found an optimal liquid PMMA formulation (2:1 liquid MMA to PMMA) for fast curing purposes. We then developed a casting procedure for the bottom layer (Figure 3.B) and demonstrated the feasibility of this approach. The observed angle measured by 3D optical profilometry matched the off-alignment angle of the centrifugation calculated geometrically (Figure 3.C). The observed angles of the PMMA layers range from $0^{\circ}$ to $13.2^{\circ}$ (center to edge well), based on a 384 well plate of size $3.175 \mathrm{~cm}$, spinning in our rotor of radius $15.24 \mathrm{~cm}$. 
A)

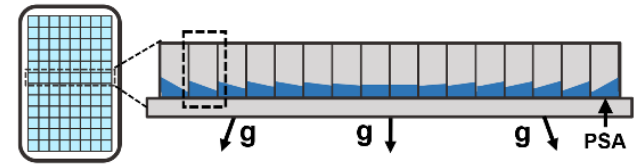

B)

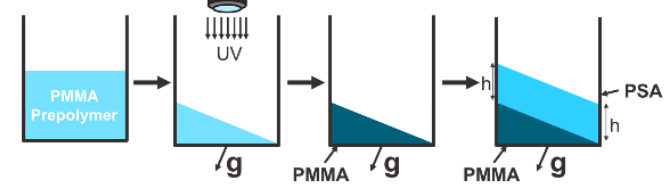

C)

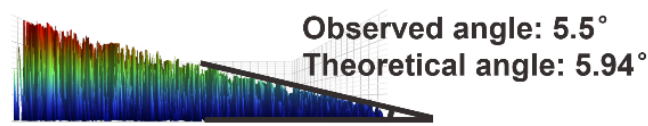

Figure 3. A) Schematic of the off-alignment effect due to the geometry of the centrifuge rotor, which causes slanted films if polymers are cast directly on the bottom of the multi-well plate. The off-aligned angle of the centrifugal force increases as the wells are further away from the center. The angles are exaggerated to illustrate the effect. B) Schematic of the UV in situ curing procedure, shown by a selected well (boxed in part A). Prepolymer of PMMA with photoinitiator and crosslinker is added to each well. The plate is then centrifuged while the prepolymer is UV cured, such that the surface of the liquid becomes perpendicular to the off-aligned centrifugal force, and the angle is preserved once the liquid solidifies upon curing. Thus, when we cast the adhesive layer under the same off-aligned centrifugal force, the relative thickness will be the same throughout the well. C) $3 \mathrm{D}$ optical profilometer image of the bottom layer in the $4^{\text {th }}$ well. Measurements show that the angle matches the calculated angle from the theory.

Together, the centrifugal deposition technique and the UV curing strategy provide a robust highthroughput thin-film preparation method, laying the groundwork for the quick, easy and quantitative centrifugal adhesion testing technique for measuring adhesive properties.

\section{Centrifugal adhesion test can differentiate formulations with various adhesive strengths}

With the centrifugal deposition technique established, we set out to develop a centrifugal adhesion screen with groundbreaking throughput, by capitalizing on the unparalleled capabilities of the centrifugation to apply homogenous force. We reasoned that we could use colored or fluorescent microparticle detachment from films at varying centrifugal speeds to measure adhesion, converting adhesive properties to optical signals for high-throughput measurement. To test this idea, we selected adhesive polymer formulations with different adhesive strengths. We used the same PSA polymer as in the previous section and modulated its adhesion by mixing it at varying ratios with non-adhesive polyethylene glycol (PEG). After depositing the polymer films using the centrifugal method and covering them with colored $325 \mu \mathrm{m}$ radius particles, we used the centrifuge to apply a pressing force on the particles to ensure good film-particle contact. We then inverted the plate and centrifuge with the particles facing outward such that they experience a centrifugal pulling force. We ramped up the centrifugal acceleration from $0 \times \mathrm{g}$ to $4700 \times \mathrm{g}$ with a $250 \times \mathrm{g}$ increment after each run, so the particles would experience increasing centrifugal force. At each centrifugal speed, we took a picture of the multi-well plate to record the detachment of particles and compare it to the picture of the original plate. We observed that for formulations with higher adhesion, particles were retained at higher angular speeds. For example, particles detached from a film composed of $70 \%$ PSA at $500 \times \mathrm{g}$, while the $90 \%$ PSA film was able to retain particles all the way up to $2750 \times \mathrm{g}$. (Figure 4 ). 


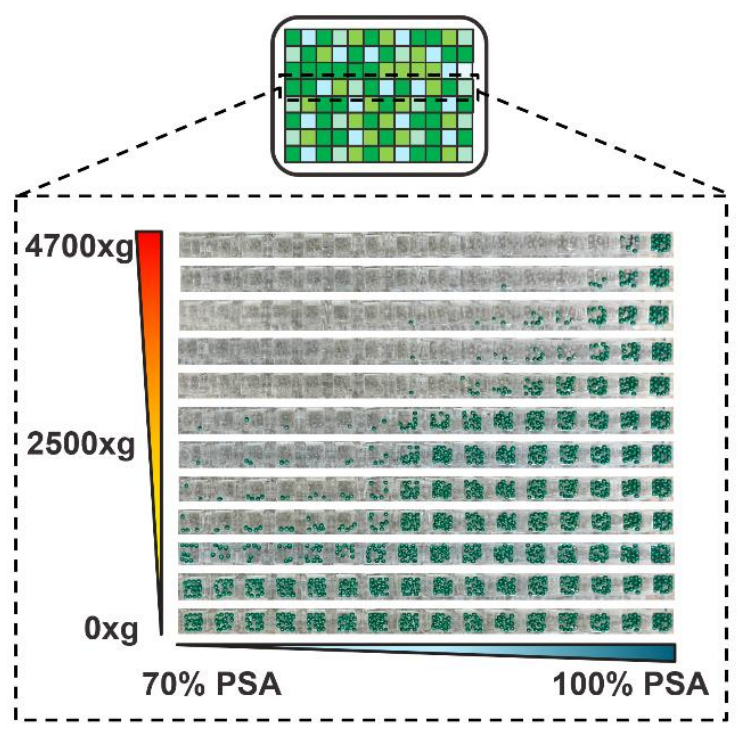

Figure 4. Pictures of the 384-well taken at different centrifugal accelerations. The same plate was centrifuged at different speeds, with a picture taken at the end of each spin.

\section{Comparison to the probe tack adhesion test validated the centrifugal adhesion test}

Next, we validated our method by comparing it to a standard, state-of-the-art measurement technique. We selected the probe tack adhesion test as the standard technique to compare, as it is widely used to characterize adhesion phenomena and mechanistically similar to our method. The probe tack test is capable of characterizing the adhesion of soft-materials with great accuracy and sensitivity, and has previously been used to measure the adhesive properties of acrylic PSAs ${ }^{33-35}$. While this test is not designed for highthroughput applications, as a single measurement can take several minutes, the axisymmetric probe test can record a detailed force vs. displacement profile while taking a video of the contact area during debonding. The probe tack test also involves using a spherical indenter in contact with an adhesive layer like our centrifugal test. Specifically, we used the same microparticles from the centrifugal test as the probe for the measurement. We next obtained $F_{\text {avg }}$ by analyzing the load-displacement profile for each of the formulations. We overlayed the results of the centrifugal adhesion testing method to the average adhesion force $\left(\mathrm{F}_{\text {avg }}\right)$ measured by the standard probe tack test.

To verify the accuracy of our method, we compared the adhesive properties of 74-100\% PSA measured by both the centrifugal adhesion test and the probe tack test. For the centrifugal adhesion test, the number of particles was counted in each well using the pictures taken. In Figure 5, we denoted the status of each well as dots of varying colors as the plate is centrifuged at successively higher speeds. Each row of dots in the figure is one centrifugation of several PSA formulations, at an angular speed that was converted to a detachment force experienced by the particle during the spin (Figure 5, left axes). While the status of each well was determined manually through visual inspection, further automation can be achieved using fluorescent particles and measuring the fluorescence with a plate reader. We repeated this procedure with red fluorescent particles (Figure S4), and we observed a similar trend as in Figure 4, demonstrating that the use of fluorescent probes enables improved automation in the workflow without any sacrifice in accuracy

The measurements from our method are in agreement with the measurements made by the probe tack test, proving that our method is capable of quantitative measurement (Figure 5.A). The best match occurs when comparing to $\mathrm{F}_{\text {avg }}$ from the axisymmetric probe test with an order one linear factor, which we found 
was 1.6. Measurements done using larger microparticles (390 $\mu$ m radius) demonstrated the robustness of this method when using particles of different sizes (Figure 5.B). The dynamic range of the probing adhesive strength can be easily tuned by simply changing the particle size in the experiment, as the centrifugal force is a function of particle radius:

$$
F=\rho_{\text {particle }} \pi r_{\text {particle }}^{3} R_{\text {rotor }} \omega^{2}
$$

where $R_{\text {rotor }}$ and $\omega$ are the rotational radius and speed, and $\rho_{\text {particle }}$ and $r_{\text {particle }}$ are the particle density and radius, respectively. Common commercially available microparticles have a size range of $\sim 10$ $1000 \mu \mathrm{m}$, which corresponds to a tunable force range of six orders of magnitude by simply changing the particle size alone. The large dynamic range promises the application of the method on a wide range of soft materials.
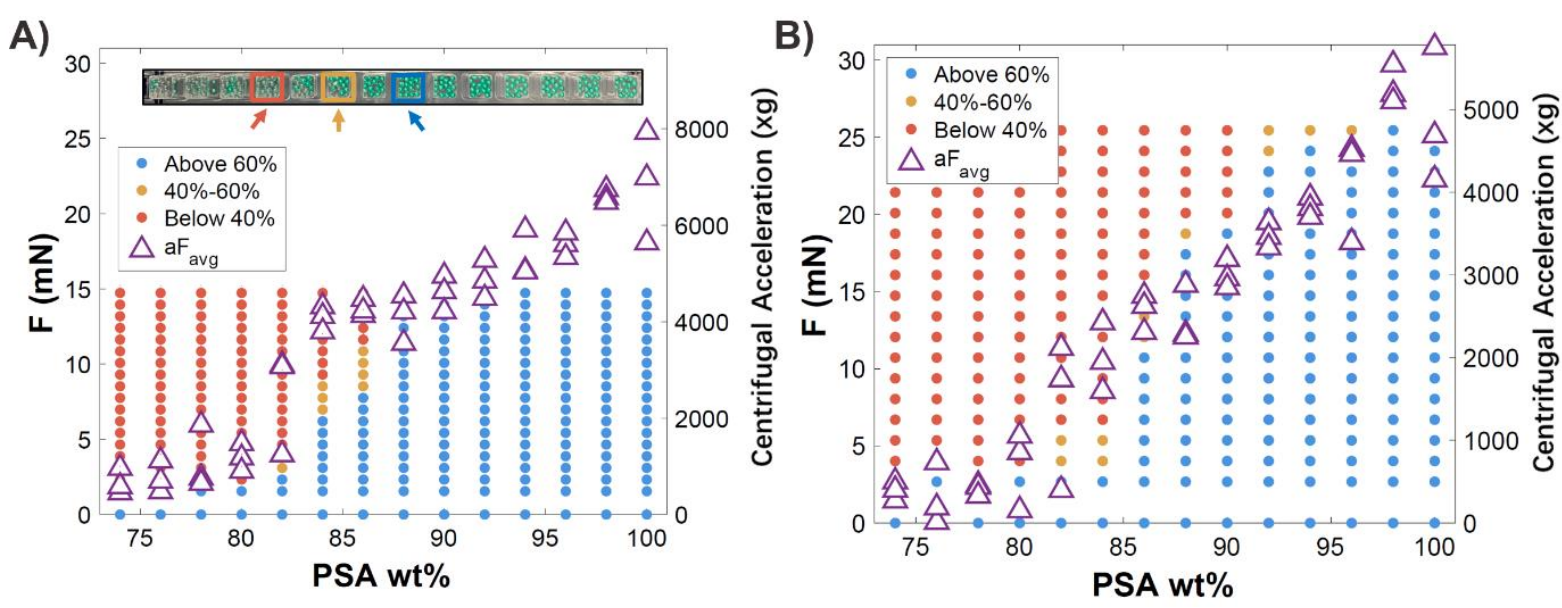

Figure 5. Comparison of measurements made by the centrifugal adhesion test and the probe tack test. The detachment of the microparticles is shown by colors. A blue dot means $>60 \%$ of the particles remained on the film, a yellow dot means $40-60 \%$ of the particles remained on the film, and a red dot means $<40 \%$ of the particles remained on the film. $F_{\text {avg }}$ measured using the probe tack test was also plotted on the same figures. A) Measurements are done by using particles of $325 \mu \mathrm{m}$ radius with the actual image of the centrifugal measurement at $750 \times \mathrm{g}$. The colored boxes and arrows indicate the detachment status of each condition B) Measurements are done by using particles of $390 \mu \mathrm{m}$ radius.

Simultaneous measurements of 384 samples showed both the throughput and the robustness of our method

For our method to be useful as a high throughput screen, we needed to ensure that there was minimal well-to-well variability across a multi-well plate. The ability to generate a pattern proves both the consistency of the measurement and the throughput of the method. With that in mind, we performed an all-plate demonstration by depositing two different PSA formulations on a well plate, pre-arranged in a pattern. The two formulations were chosen based on their adhesive strength difference, which offers enough contrast to reveal the pattern while demonstrating the sensitivity of the screen. This pattern was shown as the particles in wells containing a weaker formulation (80\% PSA) detached simultaneously at a relatively low angular speed, and while wells with a stronger formulation (92\% PSA) showed retention until much higher speeds (Figure 6.B). Furthermore, we conducted a consistency analysis on our method by calculating the Z-factor, a screening window coefficient that measures the screening assay quality and measurement data variation, with negative control (70\% PSA) and positive control (100\% PSA) deposited in alternating columns (Figure S5) ${ }^{30}$. The Z-factor increases as we ramp up the centrifugal speed, with a peak score of 0.92 at $2500 \times \mathrm{g}$. The results again indicate the robustness of the method. 
For a centrifuge rotor that holds four plates at the same time, our method has a throughput of $\mathbf{1 5 3 6}$ samples/run if using a 384-well plate. The potential throughput can be further improved by using a centrifuge rotor that can hold additional plates or doing the centrifugal test with 1536-well plates. The high throughput of this method can significantly expedite the characterization of adhesive materials, enabling large adhesive property datasets to be generated.

A)

B)
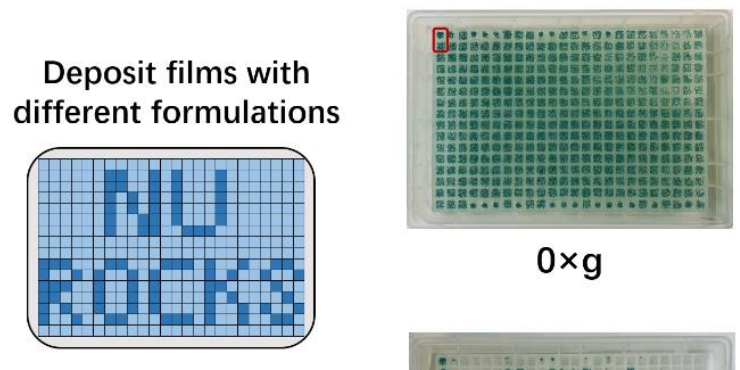

$0 \times g$

$\square$ 92\% PSA

$\square 80 \%$ PSA

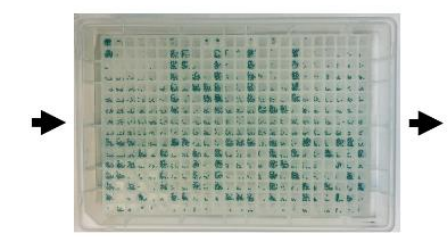

$2000 \times g$

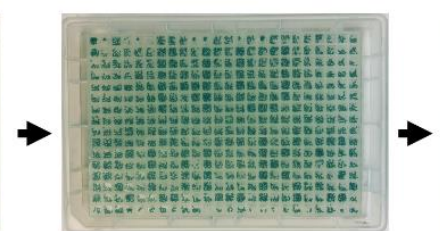

$1000 \times g$

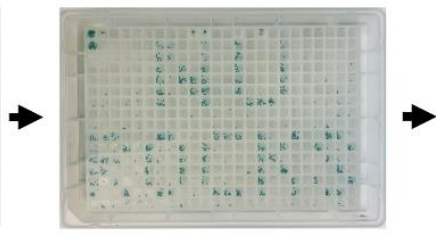

$3000 \times g$

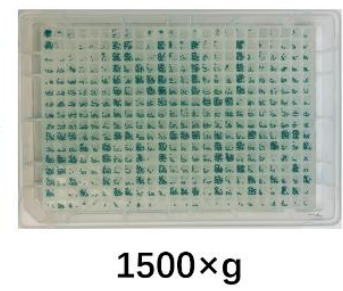

$1500 \times g$

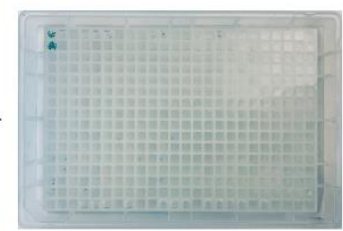

$4700 \times 9$

Figure 6. A) Schematic of the all-plate experiment to demonstrate the throughput. A pattern (dark blue) was displayed by depositing a relatively strong formulation (92\% PSA), and a weaker formulation (80\% PSA) was deposited in the background (light blue). B) Here, the particles of $325 \mu \mathrm{m}$ radius were used for the all-plate experiment. Boxed in red are two wells deposited with $100 \%$ PSA served as the negative control. The particles in the background detached first as those films are less adhesive, revealing the "NU ROCKS" pattern. The particles in the pattern eventually detached at $4700 \times \mathrm{g}$. The experiment was done following the same spin adhesion testing procedure.

\section{Conclusion}

We developed a high-throughput, fast and cost-effective centrifugal adhesion mechanical testing pipeline that can prepare and characterize adhesive materials with easily accessible laboratory equipment and consumables. The unique centrifugal deposition method overcomes the drawbacks of drop-casting, making it possible to deposit films with a smooth surface and an even thickness in all wells of a multi-well plate. Furthermore, the centrifugal adhesion testing method provides a quantitative measurement with the results validated by the standard adhesion test. We envision expanding our centrifugal force-based method to measure other mechanical properties in high throughput, expanding our capability of advanced material characterization. Our work enables the high-throughput discovery of adhesive materials and brings a novel tool to the materials community. Moreover, we hope the idea of converting mechanical properties to an optical signal can inspire additional new processes for high throughput mechanical testing.

\section{Acknowledgment}

The authors would like to thank the members of Wang lab and Tullman-Ercek lab for all the insightful discussions and supports. This work was sponsored by the MRSEC Program of the National Science Foundation under NSF Award Number DMR-1720139. This work also made use of the Keck-II facility of Northwestern University's NUANCE Center, which has received support from the SHyNE Resource (NSF ECCS-2025633), the IIN, and Northwestern's MRSEC program (NSF DMR-1720139) 


\section{References}

1. Ong, S. P. Accelerating materials science with high-throughput computations and machine learning. Comput. Mater. Sci. 161, 143-150 (2019).

2. Williams, T., McCullough, K. \& Lauterbach, J. A. Enabling catalyst discovery through machine learning and high-throughput experimentation. Chem. Mater. 32, 157-165 (2019).

3. Yamankurt, G. et al. Exploration of the nanomedicine-design space with high-throughput screening and machine learning. Nat. Biomed. Eng. 3, 318-327 (2019).

4. Mathew, K. et al. Atomate: A high-level interface to generate, execute, and analyze computational materials science workflows. Comput. Mater. Sci. 139, 140-152 (2017).

5. Malhotra, R. Combinatorial materials development. (American Chemical Society, 2002).

6. Major, J. Challenges and opportunities in high throughput screening: implications for new technologies. J. Biomol. Screen. 3, 13-17 (1998).

7. Udenfriend, S. Fluorescence assay in biology and medicine. vol. 2 (Academic Press, 2014).

8. Zhang, X. \& Xiang, Y. Combinatorial approaches for high-throughput characterization of mechanical properties. J Mater. 3, 209-220 (2017).

9. Zhao, J.-C., Jackson, M. R., Peluso, L. A. \& Brewer, L. N. A diffusion multiple approach for the accelerated design of structural materials. MRS Bull. 27, 324-329 (2002).

10. Ludwig, A., Cao, J., Brugger, J. \& Takeuchi, I. MEMS tools for combinatorial materials processing and high-throughput characterization. Meas. Sci. Technol. 16, 111-118 (2005).

11. Kim, H. J., Han, J. H., Kaiser, R., Oh, K. H. \& Vlassak, J. J. High-throughput analysis of thinfilm stresses using arrays of micromachined cantilever beams. Rev. Sci. Instrum. 79, (2008).

12. Oellers, T., Arigela, V. G., Kirchlechner, C., Dehm, G. \& Ludwig, A. Thin-Film MicrotensileTest Structures for High-Throughput Characterization of Mechanical Properties. ACS Comb. Sci. 22, 142-149 (2020).

13. Salzbrenner, B. C. et al. High-throughput stochastic tensile performance of additively manufactured stainless steel. J. Mater. Process. Technol. 241, 1-12 (2017).

14. Shull, K. R. Shull_2002-Contact mechanics and the adhesion of soft solids.pdf. vol. 36 (2002).

15. Wilke, P. et al. A direct biocombinatorial strategy toward next generation, mussel-glue inspired saltwater adhesives. J. Am. Chem. Soc. 136, 12667-12674 (2014).

16. Petrie, E. M. An introduction to adhesive and sealants. Handb. Adhes. Sealants. First ed. McGraw-Hill Prof. New York, NY, USA 2-48 (1999).

17. Dumville, J. C. et al. Dressings for the prevention of surgical site infection. Cochrane Database Syst. Rev. (2016).

18. Lee, H., Lee, B. P. \& Messersmith, P. B. A reversible wet/dry adhesive inspired by mussels and geckos. Nature 448, 338-341 (2007).

19. Autumn, K. et al. Adhesive force of a single gecko foot-hair. Nature 405, 681-685 (2000).

20. Lin, D. C., Dimitriadis, E. K. \& Horkay, F. Robust strategies for automated AFM force curve analysis-I. Non-adhesive indentation of soft, inhomogeneous materials. (2007).

21. Lin, D. C., Dimitriadis, E. K. \& Horkay, F. Robust strategies for automated AFM force curve analysis - II: adhesion-influenced indentation of soft, elastic materials. (2007).

22. Reyes, C. D. \& Garc1, J. A centrifugation cell adhesion assay for high-throughput screening of biomaterial surfaces. (2003).

23. Yang, D., Ward, A., Halvorsen, K. \& Wong, W. P. Multiplexed single-molecule force spectroscopy using a centrifuge. Nat. Commun. (2016) doi:10.1038/ncomms11026. 
24. Halvorsen, K. \& Wong, W. P. Massively Parallel Single-Molecule Manipulation Using Centrifugal Force. Biophysj 98, L53-L55 (2010).

25. Ahn, D. \& Shull, K. R. JKR studies of acrylic elastomer adhesion to glassy polymer substrates. Macromolecules 29, 4381-4390 (1996).

26. Nargang, T. M. et al. Lab on a Chip Liquid polystyrene : a room-temperature photocurable soft lithography compatible pour-and-cure-type polystyrene. 2698-2708 (2014) doi:10.1039/c4lc00045e.

27. Silvaroli, A. J. et al. Tough, Transparent, Photocurable Hybrid Elastomers. ACS Appl. Mater. Interfaces 12, 44125-44136 (2020).

28. Wang, Q. et al. Bulk and Interfacial Contributions to the Adhesion of Acrylic Emulsion-Based Pressure-Sensitive Adhesives. Macromolecules 53, 6975-6983 (2020).

29. Davis, C. S. et al. Debonding mechanisms of soft materials at short contact times. Langmuir 30, 10626-10636 (2014).

30. Zhang, J.-H., Chung, T. D. Y. \& Oldenburg, K. R. A simple statistical parameter for use in evaluation and validation of high throughput screening assays. J. Biomol. Screen. 4, 67-73 (1999).

31. Creton, C. \& Ciccotti, M. Fracture and adhesion of soft materials: A review. Reports Prog. Phys. 79, (2016).

32. Sun, J., Bao, B., He, M., Zhou, H. \& Song, Y. Recent Advances in Controlling the Depositing Morphologies of Inkjet Droplets. ACS Appl. Mater. Interfaces 7, 28086-28099 (2015).

33. Zosel, A. Adhesion and tack of polymers: Influence of mechanical properties and surface tensions. Colloid Polym. Sci. 263, 541-553 (1985).

34. Creton, C. \& Ciccotti, M. Fracture and adhesion of soft materials: A review. Reports Prog. Phys. 79, (2016).

35. Lakrout, H., Sergot, P. \& Creton, C. Direct observation of cavitation and fibrillation in a probe tack experiment on model acrylic pressure-sensitive-adhesives. J. Adhes. 69, 307-359 (1999). 